Chapter 5

\title{
The Potential Role of Neuroendocrine in Patients with Attention-Deficit/Hyperactivity Disorder
}

\author{
Liang-Jen Wang and Chih-Ken Chen \\ Additional information is available at the end of the chapter \\ http://dx.doi.org/10.5772/53609
}

\section{Introduction}

Attention-Deficit/HyperactivityDisorder (ADHD) is one of the most prevalent neurodevelopmental disorders among children. It affects 3-10\% of school-age children (Polanczyk et al., 2007), and a prevalence rate of $7.5 \%$ was reported in a local Taiwan study (Gau et al., 2005). The core symptoms of ADHD are inattention, hyperactivity, and impulsivity, and ADHD patients are commonly comorbid with other neuropsychiatric disorders, such as Oppositional Defiant Disorder (ODD), Conduct Disorder (CD), and tic disorders (American Psychiatric Association, 2000; Spencer et al., 2007). The most well-known neurobiological hypotheses to account for the complexity in etiology of ADHD are the dysregulation of catecholaminergic neurotransmission (Biederman \& Faraone, 2002). In recent years, many researchers have raised concerns with regards to the potential roles of the neuroendocrine system in the pathogenesis of ADHD (Dubrovsky, 2005; Golubchik et al., 2007; Goodyer et al., 2001; Martel et al., 2009; Strous et al., 2006), based on observations of the epidemiological data of ADHD. ADHD is more prevalent in boys than in girls, with the ratio ranging from 4 to 1 to as much as 9 to 1 , and boys generally exhibit more impaired cognitive control than girls (American Psychiatric Association, 2000). In addition, longitudinal studies have shown that there is a clear decline of symptoms with age, and a possible remission occurs after the age of 12 (Polanczyk \& Rohde, 2007). The neuroendocrine system, the activation of which is closely associated with age and gender, may influence developing neural circuitry and behavioral systems; thus it has reasonably been speculated that this system plays a role in the pathogenesis of ADHD (Martel et al., 2009).

Methylphenidate (MPH), classified as a psychostimulant, is the most widely used drug for the pharmacological management of children with ADHD (Swanson et al., 2002). The effects of $\mathrm{MPH}$ on attention result from a combination of noradrenergic and dopaminergic mecha- 
nisms (Overtoom et al., 2003; Wilens, 2008). MPH exerts treatment effects by reducing impulsivity and disruptive behavior (Huang \& Tsai, 2011), and it improves plenty of dimensions of neurocognitive function in ADHD patients (Huang et al., 2007; Pollak et al., 2010). Furthermore, some evidence has been revealed that MPH treatment possibly influences the neuroendocrine system (Hibel et al., 2007; Lurie \& O'Quinn, 1991), and these influences possibly play a role as a mediator of the therapeutic effects for ADHD patients. Therefore, we herein review the related literature which investigates the relationship between ADHD, neuroendocrine, and MPH administration.

\section{ADHD and neurosteroids}

The term "neurosteroid" was first introduced by Baulieu (1981), which indicated steroid hormones synthesized in brain cells from cholesterol, independent of peripheral endocrine sources, acted at the central nervous system. Initially, neurosteroid referred to dehydroepiandrosterone sulfate (DHEA-S) (Baulieu \& Robel, 1996). DHEA-S concentration in the brain was found to remain stable following adrenalectomy and gonadectomy. This implied that DHEA-S levels in the central neural system appeared to be independent of peripheral formation in the adrenals or gonads (Strous et al., 2006). Subsequently, progesterone, allopregnanolone, pregnenolone, dehydroepiandrosterone (DHEA) and their corresponding sulfate esters were identified as neurosteroids (Baulieu \& Robel, 1998). Neurosteroids are important substrates that have been demonstrated to affect mood expression, energy level, aggression, and general activity (Wolkowitz et al., 1999).

\subsection{DHEA and DHEA-S}

DHEA is one of the major circulating neurosteroids in human, and it is also an ACTH-regulated steroid and a substrate for the synthesis of androstenedione and testosterone (Gurnell \& Chatterjee, 2001). DHEA has been demonstrated to play several vital neurophysiological roles and to be affected by various physiological processes, including those associated with neurotrophic and neuronal excitability effects, circadian rhythms, sexual responses, immunological and stress reactions, memory, and sleep (Baulieu \& Robel, 1996; Herbert, 1998). DHEA-S is a sulfated form of DHEA that is believed to be the most abundant steroid in the body (Wolf et al., 1997). DHEA-S is measured more frequently than DHEA because circulating levels of DHEA-S are approximately 500 times higher due to its lower metabolic clearance rate and minimal diurnal variation (Longcope, 1996).

Strous et al. (2001) demonstrated that DHEA and DHEA-S levels in blood are inversely related to the severity of hyperactivity/impulsivity symptoms in children with ADHD aged between 7 and 12 years. Another study found that ADHD patients treated with methylphenidate for 3 months exhibited pre- to post-treatment increases in plasma levels of DHEA and DHEA-S for $23 \%$ and $53.6 \%$, respectively (Maayan et al., 2003). Subsequently, Lee et al. (2008) were the first research group investigating the role of neurosteroids in Asian ADHD population. They suggested that plasma DHEA-S levels in ADHD patients increased signifi- 
cantly during a 12-week MPH treatment and under a 12-week bupropion treatment as well. These studies revealed a substantial increase in DHEA levels among pre-pubertal ADHD patients; however, there was no data with regards to age- and gender-matched healthy controls for comparison. Finally, our research team demonstrated that salivary DHEA levels were significantly lower in ADHD patients than those in healthy controls (Wang et al., 2011b). Salivary DHEA levels were not significantly correlated with ADHD clinical symptom severity, but positively correlated with performance in a neuropsychological test (Conner's Continuous Performance Test, CPT). Thus the authors suggested that lower morning DHEA levels might be a biological laboratory marker for ADHD, particularly for performance during CPT. In the longitudinal analyses, we found that morning salivary DHEA levels significantly increased under the 6-month MPH treatment course for ADHD patients. However, the salivary DHEA levels in healthy age- and gender- matched controls remain unchanged during the 6-month natural observation. Similar with the findings in the crosssectional survey, DHEA levels exhibited a significant and independent association with overall CPT performance during the course of MPH treatment (Wang et al., 2011a). We could thus determine that the elevation of DHEA levels among ADHD patients is not derived from natural physiological change, but from $\mathrm{MPH}$ administration.

There is interesting coincidence in the similarity of the natural course of ADHD, brain development and age-related change in DHEA/DHEA-S levels. ADHD symptoms generally decline in severity between puberty and the early twenties (Biederman et al., 2000). Some brain imaging studies have found there to be a dysfunction of the cerebellar-striatal-prefrontal circuitry in ADHD (Gogtay et al., 2002), and that this may be the result of delayed maturation of the cerebral cortex, especially the prefrontal region (Shaw et al., 2007; McAlonan et al., 2009). Plasma DHEA/DHEA-S levels change with age, being low in the first years of life, then rapidly increasing from about eight years of age through puberty to reach their highest levels during early adulthood (de Peretti \& Forest, 1978). DHEA-S has antiamnestic effects, and also anxiolytic and anti-aggressive properties (Wolf \& Kirschbaum, 1999). Both DHEA and DHEA-S have been shown to regulate the motility and growth of neocortical neurons in the rodent brain (Compagnone \& Mellon, 1998). This implies that DHEA/DHEA-S exert biological actions that may play crucial roles in guiding cortical projections to appropriate targets, and thus may be important for the regulation of neurodevelopment (Golubchik et al., 2007). It has been proposed that DHEA/DHEA-S exerts its positive effects in ADHD patients through stimulatory or antagonist effects at the gammaaminobutyric acid $A\left(\mathrm{GABA}_{\mathrm{A}}\right)$ receptor and facilitation of the N-methyl-D-aspartate (NMDA) activity (Davies et al., 2009; Strous et al., 2001; Tang et al., 1999). DHEA protects hippocampal neuronal activity from glutamate toxicity. DHEA-S also protects hippocampal neuronal cells from excitatory amino acid induced neurotoxicity. Taken together, DHEA and DHEA-S may provide an important antioxidant function and may thus play a role in neurodevelopment and have neuroprotective effects (Strous et al., 2006).

Some molecular genetic studies demonstrated candidate genes contributing to ADHD. The steroid sulfatase (STS) gene, which escapes X inactivation in humans, desulfates several sulfatedsteroids, including DHEA-S to DHEA. In an animal study, it has been noticed that the 
STS may modify the attentional function and motor impulsivity through administration of the substrate DHEA and DHEA-S (Davies et al., 2009). The same research team showed that the $39, \mathrm{X}\left(\mathrm{Y}^{*}\right) \mathrm{O}$ mice (which lack the STS gene but no other known genes as a consequence of end-to-end fusion of the $X$ and $Y$ chromosomes) exhibited significantly lower DHEA serum levels than 40,XY mice. They concluded that STS-deficient mice exhibit endophenotypes relevant to ADHD (Trent et al., 2012). In human studies, Kent et al. (2008) have demonstrated that boys with X-linked ichthyosis who have a deletion or point mutation of STS are at an increased risk of ADHD. In addition, Brookes et al. (2008) indicated that common variants within the STS gene may increase susceptibility to ADHD. The over transmitted risk allele of rs12861247 was also associated with reduced STS mRNA expression, and hence deficit in STS protein production is at a significantly increased risk of developing ADHD (Brookes et al., 2010). However, ethnic differences in epidemiology and genetic polymorphism of ADHD patients have been demonstrated in some studies (Nikolaidis \& Gray, 2009; Pastor \& Reuben, 2005). It remains unclear whether the relationships between the STS gene, ADHD and neurosteroids also exist among non-Caucasian populations.

Neurochemical findings showing MPH exerts its effects on catecholamines in areas such as the prefrontal cortex, nucleus accumbens, and striatum are consistent with the neurobiological and clinical effects of MPH on memory, attention, and movement (Wilens, 2008). The neuroendocrine effects of $\mathrm{MPH}$ administration might be related to its dopaminergic and adrenergic agonistic activity (Hibel et al., 2007; Lurie \& O'Quinn, 1991; Weizman et al., 1987). Hibel et al. (2007) demonstrated that children taking concerta (methylphenidate extended-release tablets) had higher average levels of DHEA than those who were non-medicated. Several studies suggested that MPH increased DHEA or DHEA-S levels of ADHD patients during a 3-month treatment (Lee et al., 2008; Maayan et al., 2003; Wang et al., 2011a). One possible neurochemical explanation for this phenomenon is that MPH-induced increases in DHEA or DHEA-S may act either by decreasing levels of the $\mathrm{GABA}_{\mathrm{A}}$ antagonist-like pregnenolone sulfate, or by increasing levels of the $\mathrm{GABA}_{\mathrm{A}}$ agonist-like progesterone metabolites (Robel \& Baulieu, 1995). In addition, the direct influence of medications on salivary hormones may act on the secretion and feedback control of the HPA and hypothalamic-pituitary-gonadal (HPG) axes. MPH may also indirectly influence DHEA or DHEA-S by attenuating or potentiating the impact of environmental events and subjective experience on HPA axis and HPG activity (Hibel et al., 2007). However, it is not clear whether DHEA or DHEA-S exerts effects in conjunction with or independently of MPH on neurocognitive function in ADHD. It remains to be clarified whether DHEA or DHEA-S plays a role as a mediator of the therapeutic effects of $\mathrm{MPH}$, or if these associations are epiphenomena of the benefits of MPH treatment. Moreover, it would also be interesting to investigate whether DHEA or DHEA-S could directly benefit the treatment of ADHD patients.

\subsection{Other neurosteroids and gonadal hormones}

Neurosteroids, other than DHEA and DHEA-S, mainly contain pregnenolone (PREG) and progesterone, which is metabolized to $5 a$-dihydroprogesterone ( $5 \alpha$-DH PROG) and $3 \alpha, 5 \alpha-$ tetrahydroprogesterone ( $3 \alpha, 5 \alpha-\mathrm{TH}$ PROG), also named allopregnanolone (Vallee et al., 
2001). PREG is the principal precursor of DHEA. The sulfated form of pregnenolone (PREGS) exerts its neurochemical action as a negative modulator of the $\mathrm{GABA}_{\mathrm{A}}$ and also as a positive modulator of the NMDA subtype of glutamate receptor (Mayo et al., 2001). PREG-S is also capable of modulating acetylcholine neurotransmission associated with paradoxical sleep modifications (Mayo et al., 2003). In animal studies, PREG-S in the hippocampus region plays a physiological role in preserving and enhancing cognitive abilities, possibly via an interaction with central cholinergic systems (Vallee et al., 1997; Vallee et al., 2001). Among humans, PREG appears to improve clinical symptoms in patients with mood disorders (Osuji et al., 2010), and in patients with schizophrenia as well (Marx et al., 2011). To date, there has been only one study investigating the relationship of PREG and ADHD (Strous et al., 2001), and this study revealed that PREG levels in the blood are inversely related to the severity of hyperactivity/impulsivity symptoms in children with ADHD.

Gonadal hormones may act on the prenatal development of dopaminergic neural circuitry and dopamine function in the nucleus accumbens, striatum, and prefrontal cortex via its masculinizing effects (Martel et al., 2009). Therefore, gonadal hormones may modulate corresponding deficits in cognitive control and reward processes in ADHD. In animal studies, estradiol and progesterone both appear capable of inducing increases in dendritic spine density during development. Estradiol and progesterone have been also proposed to play a role in ADHD, because they are synthesized de novo in the cerebellum during critical developmental periods (Dean \& McCarthy, 2008). In addition, estrogen has been identified to have neuroprotective effects through protection against oxidative stress, and neurotrophic cross-talk through the signal cascade shared with neurotrophic factors (Sawada \& Shimohama, 2000). However, the relationship of estradiol, progesterone and ADHD in humans has not yet been well-established in clinical studies.

Prenatal testosterone exposure has been implied in the etiology of ADHD. High levels of prenatal testosterone may moderate the relationship between prenatal risk factors, and further affect dopaminergic neural circuitry by slowing down neural development globally (Morris et al., 2004). Several studies have examined the association between the presence of ADHD phenotype and the ratio of the length of the second and fourth digits (2D:4D ratio), which is a marker of fetal testosterone exposure (de Bruin et al., 2006; Lemiere et al., 2010; Stevenson et al., 2007). However, the findings are inconsistent between these studies. Furthermore, Yu \& Shi (2009) found that salivary testosterone levels are higher in children with aggressive tendencies than those without aggressive tendencies. Whereas Dorn et al., (2009) suggested that no significant difference in salivary testosterone levels between children with and without disruptive behavior disorders. Regarding the effects of MPH on testosterone, Avital et al., (2011) indicated that long-term exposure to MPH led to elevated testosterone levels in rodents. However, Mattison et al., (2011) reported that MPH administration in rhesus macaques, beginning before puberty, led to delay in pubertal testicular development until 5 years of age. Among humans, Hibel et al. (2007) investigated the relationship of salivary biomarker levels, diurnal variation and the effects of medications among maltreated or lowincome disadvantaged children. They found that testosterone in non-medicated children decreased along with time in a day, but those in children taking psychostimulants remained 
unchanged. In summary, evidence about the role of gonadal hormones in the aetiology of ADHD in humans is remains scarce, thus future clinical studies are warranted to elucidate this issue.

\section{Cortisol}

Dysfunction of the hypothalamic-pituitary-adrenal (HPA) axis in ADHD children was proposed to address the complexity in the pathophysiology of ADHD (Kaneko et al., 1993; Ma et al., 2011). The HPA axis plays an important role in regulating central nervous system neurotransmitters and behavior, such as attention, emotion, memory, and learning (Talge et al., 2007). The function of the HPA axis has typically been assessed by cortisol levels, which can be measured from a number of sources (saliva, urine, and blood plasma) (Hellhammer et al., 2009). Low cortisol levels generally reflect under arousal or an elevated threshold for the detection of stressors (Freitag et al., 2009; Kaneko et al., 1993). Many clinical studies investigated cortisol levels in either a stress response or an awakening response (Freitag et al., 2009; Popma et al., 2006; Stadler et al., 2011; Yang et al., 2007). For example, Popma et al. (2006) reported that patients with disruptive behavior disorders showed a significantly decreased cortisol response after a standard public speaking task as compared with the normal controls. Yang et al. (2007) demonstrated that the magnitude of the increase in cortisol reactivity to stress was inversely correlated with aggression tendency in patients with ADHD. Freitag et al. (2009) indicated that ADHD children comorbid with ODD showed a weaker cortisol awakening response compared to ADHD children without comorbidity and control children. Stadler et al. (2011) reported that ADHD patients scoring high on callous unemotional traits showed a blunted HPA axis reactivity to the experimentally induced stress. Moreover, it has also been suggested that the cortisol reactivity to stress at baseline in ADHD patients could predict treatment effects (van de Wiel et al., 2004), and was associated with the oneyear outcome (King et al., 1998).

On the other hand, some studies aimed to determine the basal level of morning cortisol in ADHD patients, and to determine the relationship between cortisol levels and ADHD related social/behavioral symptoms or cognition deficit. Among these, Schulz et al., (1997) showed that there was no significant difference in basal cortisol levels between aggressive and nonaggressive boys with ADHD. In addition, the basal level of morning cortisol was not significantly correlated with the severity of ADHD hyperactivity/impulsivity symptoms (Strous et al., 2001) and the performance in neuropsychological test (Wang et al., 2011b). However, there is one study which demonstrated gender differences in the cortisol levels among a community sample of adolescents, which showed that females carry a positive and males a negative association between cortisol and conduct symptoms (Young et al., 2012). For ADHD subtypes, Ma et al. (2011) reported that the level of cortisol in the ADHD group was significantly lower than that of the control group. There was a significant difference in cortisol levels between ADHD subtypes: The level of cortisol of the ADHD-predominantly hyperactive impulsive type was significantly lower than that of ADHD-predominantly inattention type and ADHD-combined type (Ma et al., 2011). Fur- 
thermore, a recent meta-analysis revealed that the age of the children significantly moderated the relation between basal cortisol and externalizing behavior. Externalizing behavior was associated with higher basal cortisol in preschoolers, and with lower basal cortisol in elementary school-aged children (Alink et al., 2008).

Regarding with the effects of MPH on cortisol, Weizman et al. (1987) reported that plasma cortisol levels of ADHD patients increased under acute challenge of MPH; nevertheless, the effects disappeared when subjects were re-challenged after 4 weeks. Similar effect of acute challenge MPH for increasing cortisol levels was also observed among normal adults (Joyce et al., 1986). However, Lee et al. (2008) showed that during a 12-week period, there was no significant change in cortisol levels in ADHD patients under MPH treatment. In contrast, Chen et al. (2012) reported that basal plasma cortisol levels were significant increased after treatment either with MPH or with atomoxetine. Furthermore, our research team showed that the morning levels of salivary cortisol in the patients with ADHD were significantly increased from baseline at 1 month after the MPH treatment was started (Wang et al., 2012). Subsequently, the cortisol levels dropped to an intermediary level that differed from the baseline and 1-month values. The effects of MPH on the neuroendocrine system were proposed to result from a combination of noradrenergic and dopaminergic mechanisms (Lurie \& O'Quinn, 1991). The higher concentrations of dopamine and norepinephrine could promote the release of corticotropin releasing hormone $(\mathrm{CRH})$ and the adrenocorticotropic hormone (ACTH) (Biondi \& Picardi, 1999). It might be a possible explanation of MPH-induced cortisol elevation; however, the effect of MPH on cortisol secretion seems to be temporary. Acute tolerance to $\mathrm{MPH}$ has long been reported in the treatment of ADHD in children (Swanson et al., 1999). It warrants further investigation to clarify whether the transient effect on cortisol increment shares similar neurobiological mechanisms with the tolerance of $\mathrm{MPH}$.

\section{Conclusion}

Awareness of the prominent position that the neuroendocrine system has to play in pathophysiology of ADHD is increasing. DHEA and DHEA-S are important neurosteroids substrates which demonstrate a potential correlation to symptoms severity and neurocognitive function in ADHD patients. MPH, the most therapeutically efficient drugs in

ADHD, exerts its pharmacological effects via increasing the level of the dopamine and norepinephrine. Extant studies almost identically indicate that MPH administration would lead to increases in the levels of DHEA or DHEA-S. With regards to other neurosteroids or gonadal hormones, their influence on developing neural circuitry and behavioral systems has already been established in animal models. However, many findings noted in animal studies have yet to be replicated in humans, in particular patients with ADHD. The HPA-axis dysfunction, which was measured by cortisol levels, has also been indicated to address the complexity in the pathogenesis of ADHD. Current studies revealed that ADHD patients, especially for those with higher aggression tendencies or comorbidities, might have lower levels of cortisol than healthy controls. MPH administration is able to elevate cortisol levels, 
but the effects seem to be temporary. To date, much remains unclear about the complex interaction between neuroendocrine system, pathophysiology of ADHD and effects of MPH. Further research at the basic scientific level as well as in the context of double-blinded placebo controlled investigation is mandated to better elucidate the role of neuroendocrine in the understanding and management of ADHD patients.

\section{Author details}

Liang-Jen Wang ${ }^{1,2}$ and Chih-Ken Chen ${ }^{2,3}$

1 Department of Child and Adolescent Psychiatry, Chang Gung Memorial Hospital - Kaohsiung Medical Center, Chang Gung University College of Medicine, Kaohsiung, Taiwan

2 Chang Gung University School of Medicine, Taoyuan, Taiwan

3 Department of Psychiatry, Chang Gung Memorial Hospital, Keelung, Taiwan

\section{References}

[1] Alink, L.R., Van Ijzendoorn, M.H., Bakermans-Kranenburg, M.J., Mesman, J., Juffer, F. \& Koot, H.M. (2008). Cortisol and externalizing behavior in children and adolescents: mixed meta-analytic evidence for the inverse relation of basal cortisol and cortisol reactivity with externalizing behavior. Dev Psychobiol, 50, 427-450.

[2] American Psychiatric Association (2000). Diagnostic and Statistical Manual of Mental disorders. American Psychiatric Association, Washington, DC.

[3] Avital, A., Dolev, T., Aga-Mizrachi, S. \& Zubedat, S. (2011). Environmental enrichment preceding early adulthood methylphenidate treatment leads to long term increase of corticosterone and testosterone in the rat. PLoS One, 6, e22059.

[4] Baulieu, E.E. \& Robel, P. (1996). Dehydroepiandrosterone and dehydroepiandrosterone sulfate as neuroactive neurosteroids. J Endocrinol, 150 Suppl, S221-239.

[5] Baulieu, E.E. \& Robel, P. (1998). Dehydroepiandrosterone (DHEA) and dehydroepiandrosterone sulfate (DHEAS) as neuroactive neurosteroids. Proc Natl Acad Sci U S A, 95, 4089-4091.

[6] Biederman, J. \& Faraone, S.V. (2002). Current concepts on the neurobiology of Attention-Deficit/Hyperactivity Disorder. J Atten Disord, 6 Suppl 1, S7-16.

[7] Biederman, J., Mick, E. \& Faraone, S.V. (2000). Age-dependent decline of symptoms of attention deficit hyperactivity disorder: impact of remission definition and symptom type. Am J Psychiatry, 157, 816-818. 
[8] Biondi, M. \& Picardi, A. (1999). Psychological stress and neuroendocrine function in humans: the last two decades of research. Psychother Psychosom, 68, 114-150.

[9] Brookes, K.J., Hawi, Z., Kirley, A., Barry, E., Gill, M. \& Kent, L. (2008). Association of the steroid sulfatase (STS) gene with attention deficit hyperactivity disorder. Am J Med Genet B Neuropsychiatr Genet, 147B, 1531-1535.

[10] Brookes, K.J., Hawi, Z., Park, J., Scott, S., Gill, M. \& Kent, L. (2010). Polymorphisms of the steroid sulfatase (STS) gene are associated with attention deficit hyperactivity disorder and influence brain tissue mRNA expression. Am J Med Genet B Neuropsychiatr Genet, 153B, 1417-1424.

[11] Chen, Y.H., Lin, X.X., Chen, H., Liu, Y.Y., Lin, G.X., Wei, L.X., et al. (2012). The change of the cortisol levels in children with ADHD treated by methylphenidate or atomoxetine. J Psychiatr Res, 46, 415-416.

[12] Compagnone, N.A. \& Mellon, S.H. (1998). Dehydroepiandrosterone: a potential signalling molecule for neocortical organization during development. Proc Natl Acad Sci U S A, 95, 4678-4683.

[13] Davies, W., Humby, T., Kong, W., Otter, T., Burgoyne, P.S. \& Wilkinson, L.S. (2009). Converging pharmacological and genetic evidence indicates a role for steroid sulfatase in attention. Biol Psychiatry, 66, 360-367.

[14] De Bruin, E.I., Verheij, F., Wiegman, T. \& Ferdinand, R.F. (2006). Differences in finger length ratio between males with autism, pervasive developmental disorder-not otherwise specified, ADHD, and anxiety disorders. Dev Med Child Neurol, 48, 962-965.

[15] De Peretti, E. \& Forest, M.G. (1978). Pattern of plasma dehydroepiandrosterone sulfate levels in humans from birth to adulthood: evidence for testicular production. $J$ Clin Endocrinol Metab, 47, 572-577.

[16] Dean, S.L. \& Mccarthy, M.M. (2008). Steroids, sex and the cerebellar cortex: implications for human disease. Cerebellum, 7, 38-47.

[17] Dorn, L.D., Kolko, D.J., Susman, E.J., Huang, B., Stein, H., Music, E., et al. (2009). Salivary gonadal and adrenal hormone differences in boys and girls with and without disruptive behavior disorders: Contextual variants. Biol Psychol, 81, 31-39.

[18] Dubrovsky, B.O. (2005). Steroids, neuroactive steroids and neurosteroids in psychopathology. Prog Neuropsychopharmacol Biol Psychiatry, 29, 169-192

[19] Freitag, C.M., Hanig, S., Palmason, H., Meyer, J., Wust, S. \& Seitz, C. (2009). Cortisol awakening response in healthy children and children with ADHD: impact of comorbid disorders and psychosocial risk factors. Psychoneuroendocrinology, 34, 1019-1028.

[20] Gau, S.S., Chong, M.Y., Chen, T.H. \& Cheng, A.T. (2005). A 3-year panel study of mental disorders among adolescents in Taiwan. Am J Psychiatry, 162, 1344-1350. 
[21] Gogtay, N., Giedd, J. \& Rapoport, J.L. (2002). Brain development in healthy, hyperactive, and psychotic children. Arch Neurol, 59, 1244-1248.

[22] Golubchik, P., Lewis, M., Maayan, R., Sever, J., Strous, R. \& Weizman, A. (2007). Neurosteroids in child and adolescent psychopathology. Eur Neuropsychopharmacol, 17, 157-164.

[23] Goodyer, I.M., Park, R.J., Netherton, C.M. \& Herbert, J. (2001). Possible role of cortisol and dehydroepiandrosterone in human development and psychopathology. $\mathrm{Br} J$ Psychiatry, 179, 243-249.

[24] Gurnell, E.M. \& Chatterjee, V.K. (2001). Dehydroepiandrosterone replacement therapy. Eur J Endocrinol, 145, 103-106.

[25] Hellhammer, D.H., Wust, S. \& Kudielka, B.M. (2009). Salivary cortisol as a biomarker in stress research. Psychoneuroendocrinology, 34, 163-171.

[26] Herbert, J. (1998). Neurosteroids, brain damage, and mental illness. Exp Gerontol, 33, 713-727.

[27] Hibel, L.C., Granger, D.A., Cicchetti, D. \& Rogosch, F. (2007). Salivary biomarker levels and diurnal variation: associations with medications prescribed to control children's problem behavior. Child Dev, 78, 927-937.

[28] Huang, Y.S., Chao, C.C., Wu, Y.Y., Chen, Y.Y. \& Chen, C.K. (2007). Acute effects of methylphenidate on performance during the Test of Variables of Attention in children with attention deficit/hyperactivity disorder. Psychiatry Clin Neurosci, 61, 219-225.

[29] Huang, Y.S. \& Tsai, M.H. (2011). Long-term outcomes with medications for attention-deficit hyperactivity disorder: current status of knowledge. CNS Drugs, 25, 539-554.

[30] Joyce, P.R., Donald, R.A., Nicholls, M.G., Livesey, J.H. \& Abbott, R.M. (1986). Endocrine and behavioral responses to methylphenidate in normal subjects. Biol Psychiatry, 21, 1015-1023.

[31] Kaneko, M., Hoshino, Y., Hashimoto, S., Okano, T. \& Kumashiro, H. (1993). Hypothalamic-pituitary-adrenal axis function in children with attention-deficit hyperactivity disorder. J Autism Dev Disord, 23, 59-65.

[32] Kent, L., Emerton, J., Bhadravathi, V., Weisblatt, E., Pasco, G., Willatt, L.R., et al. (2008). X-linked ichthyosis (steroid sulfatase deficiency) is associated with increased risk of attention deficit hyperactivity disorder, autism and social communication deficits. J Med Genet, 45, 519-524.

[33] King, J.A., Barkley, R.A. \& Barrett, S. (1998). Attention-deficit hyperactivity disorder and the stress response. Biol Psychiatry, 44, 72-74. 
[34] Lee, M.S., Yang, J.W., Ko, Y.H., Han, C., Kim, S.H., Joe, S.H., et al. (2008). Effects of methylphenidate and bupropion on DHEA-S and cortisol plasma levels in attentiondeficit hyperactivity disorder. Child Psychiatry Hum Dev, 39, 201-209.

[35] Lemiere, J., Boets, B. \& Danckaerts, M. (2010). No association between the 2D:4D fetal testosterone marker and multidimensional attentional abilities in children with ADHD. Dev Med Child Neurol, 52, e202-208.

[36] Longcope, C. (1996). Dehydroepiandrosterone metabolism. J Endocrinol, 150 Suppl, S125-127.

[37] Lurie, S. \& O'quinn, A. (1991). Neuroendocrine responses to methylphenidate and damphetamine: applications to attention-deficit disorder. J Neuropsychiatry Clin Neurosci, 3, 41-50.

[38] Ma, L., Chen, Y.H., Chen, H., Liu, Y.Y. \& Wang, Y.X. (2011). The function of hypothalamus-pituitary-adrenal axis in children with ADHD. Brain Res, 1368, 159-162.

[39] Maayan, R., Yoran-Hegesh, R., Strous, R., Nechmad, A., Averbuch, E., Weizman, A., et al. (2003). Three-month treatment course of methylphenidate increases plasma levels of dehydroepiandrosterone (DHEA) and dehydroepiandrosterone-sulfate (DHEA-S) in attention deficit hyperactivity disorder. Neuropsychobiology, 48, 111-115.

[40] Martel, M.M., Klump, K., Nigg, J.T., Breedlove, S.M. \& Sisk, C.L. (2009). Potential hormonal mechanisms of attention-deficit/hyperactivity disorder and major depressive disorder: a new perspective. Horm Behav, 55, 465-479.

[41] Marx, C.E., Bradford, D.W., Hamer, R.M., Naylor, J.C., Allen, T.B., Lieberman, J.A., et al. (2011). Pregnenolone as a novel therapeutic candidate in schizophrenia: emerging preclinical and clinical evidence. Neuroscience, 191, 78-90.

[42] Mattison, D.R., Plant, T.M., Lin, H.M., Chen, H.C., Chen, J.J., Twaddle, N.C., et al. (2011). Pubertal delay in male nonhuman primates (Macaca mulatta) treated with methylphenidate. Proc Natl Acad Sci U S A, 108, 16301-16306.

[43] Mayo, W., George, O., Darbra, S., Bouyer, J.J., Vallee, M., Darnaudery, M., et al. (2003). Individual differences in cognitive aging: implication of pregnenolone sulfate. Prog Neurobiol, 71, 43-48.

[44] Mayo, W., Le Moal, M. \& Abrous, D.N. (2001). Pregnenolone sulfate and aging of cognitive functions: behavioral, neurochemical, and morphological investigations. Horm Behav, 40, 215-217.

[45] Mcalonan, G.M., Cheung, V., Chua, S.E., Oosterlaan, J., Hung, S.F., Tang, C.P., et al. (2009). Age-related grey matter volume correlates of response inhibition and shifting in attention-deficit hyperactivity disorder. Br J Psychiatry, 194, 123-129.

[46] Morris, J.A., Jordan, C.L. \& Breedlove, S.M. (2004). Sexual differentiation of the vertebrate nervous system. Nat Neurosci, 7, 1034-1039. 
[47] Nikolaidis, A. \& Gray, J.R. (2009). ADHD and the DRD4 exon III 7-repeat polymorphism: an international meta-analysis. Soc Cogn Affect Neurosci.

[48] Osuji, I.J., Vera-Bolanos, E., Carmody, T.J. \& Brown, E.S. (2010). Pregnenolone for cognition and mood in dual diagnosis patients. Psychiatry Res, 178, 309-312.

[49] Overtoom, C.C., Verbaten, M.N., Kemner, C., Kenemans, J.L., Van Engeland, H., Buitelaar, J.K., et al. (2003). Effects of methylphenidate, desipramine, and L-dopa on attention and inhibition in children with Attention Deficit Hyperactivity Disorder. Behav Brain Res, 145, 7-15.

[50] Pastor, P.N. \& Reuben, C.A. (2005). Racial and ethnic differences in ADHD and LD in young school-age children: parental reports in the National Health Interview Survey. Public Health Rep, 120, 383-392.

[51] Polanczyk, G., De Lima, M.S., Horta, B.L., Biederman, J. \& Rohde, L.A. (2007). The worldwide prevalence of ADHD: a systematic review and metaregression analysis. Am J Psychiatry, 164, 942-948.

[52] Polanczyk, G. \& Rohde, L.A. (2007). Epidemiology of attention-deficit/hyperactivity disorder across the lifespan. Curr Opin Psychiatry, 20, 386-392.

[53] Pollak, Y., Shomaly, H.B., Weiss, P.L., Rizzo, A.A. \& Gross-Tsur, V. (2010). Methylphenidate effect in children with ADHD can be measured by an ecologically valid continuous performance test embedded in virtual reality. CNS Spectr, 15, 125-130.

[54] Popma, A., Jansen, L.M., Vermeiren, R., Steiner, H., Raine, A., Van Goozen, S.H., et al. (2006). Hypothalamus pituitary adrenal axis and autonomic activity during stress in delinquent male adolescents and controls. Psychoneuroendocrinology, 31, 948-957.

[55] Robel, P. \& Baulieu, E.E. (1995). Neurosteroids: biosynthesis and function. Crit Rev Neurobiol, 9, 383-394.

[56] Sawada, H. \& Shimohama, S. (2000). Neuroprotective effects of estradiol in mesencephalic dopaminergic neurons. Neurosci Biobehav Rev, 24, 143-147.

[57] Schulz, K.P., Halperin, J.M., Newcorn, J.H., Sharma, V. \& Gabriel, S. (1997). Plasma cortisol and aggression in boys with ADHD. J Am Acad Child Adolesc Psychiatry, 36, 605-609.

[58] Shaw, P., Eckstrand, K., Sharp, W., Blumenthal, J., Lerch, J.P., Greenstein, D., et al. (2007). Attention-deficit/hyperactivity disorder is characterized by a delay in cortical maturation. Proc Natl Acad Sci U S A, 104, 19649-19654.

[59] Spencer, T.J., Biederman, J. \& Mick, E. (2007). Attention-deficit/hyperactivity disorder: diagnosis, lifespan, comorbidities, and neurobiology. J Pediatr Psychol, 32, 631-642.

[60] Stadler, C., Kroeger, A., Weyers, P., Grasmann, D., Horschinek, M., Freitag, C., et al. (2011). Cortisol reactivity in boys with attention-deficit/hyperactivity disorder and 
disruptive behavior problems: The impact of callous unemotional traits. Psychiatry Res, 187, 204-209.

[61] Stevenson, J.C., Everson, P.M., Williams, D.C., Hipskind, G., Grimes, M. \& Mahoney, E.R. (2007). Attention deficit/hyperactivity disorder (ADHD) symptoms and digit ratios in a college sample. Am J Hum Biol, 19, 41-50.

[62] Strous, R.D., Maayan, R. \& Weizman, A. (2006). The relevance of neurosteroids to clinical psychiatry: from the laboratory to the bedside. Eur Neuropsychopharmacol, 16, 155-169.

[63] Strous, R.D., Spivak, B., Yoran-Hegesh, R., Maayan, R., Averbuch, E., Kotler, M., et al. (2001). Analysis of neurosteroid levels in attention deficit hyperactivity disorder. Int J Neuropsychopharmacol, 4, 259-264.

[64] Swanson, J., Gupta, S., Guinta, D., Flynn, D., Agler, D., Lerner, M., et al. (1999). Acute tolerance to methylphenidate in the treatment of attention deficit hyperactivity disorder in children. Clin Pharmacol Ther, 66, 295-305.

[65] Swanson, J.M., Gupta, S., Williams, L., Agler, D., Lerner, M. \& Wigal, S. (2002). Efficacy of a new pattern of delivery of methylphenidate for the treatment of ADHD: effects on activity level in the classroom and on the playground. J Am Acad Child Adolesc Psychiatry, 41, 1306-1314.

[66] Talge, N.M., Neal, C. \& Glover, V. (2007). Antenatal maternal stress and long-term effects on child neurodevelopment: how and why? J Child Psychol Psychiatry, 48, 245-261.

[67] Tang, Y.P., Shimizu, E., Dube, G.R., Rampon, C., Kerchner, G.A., Zhuo, M., et al. (1999). Genetic enhancement of learning and memory in mice. Nature, 401, 63-69.

[68] Trent, S., Dennehy, A., Richardson, H., Ojarikre, O.A., Burgoyne, P.S., Humby, T., et al. (2012). Steroid sulfatase-deficient mice exhibit endophenotypes relevant to attention deficit hyperactivity disorder. Psychoneuroendocrinology, 37, 221-229.

[69] Vallee, M., Mayo, W., Darnaudery, M., Corpechot, C., Young, J., Koehl, M., et al. (1997). Neurosteroids: deficient cognitive performance in aged rats depends on low pregnenolone sulfate levels in the hippocampus. Proc Natl Acad Sci U S A, 94, 14865-14870.

[70] Vallee, M., Mayo, W. \& Le Moal, M. (2001). Role of pregnenolone, dehydroepiandrosterone and their sulfate esters on learning and memory in cognitive aging. Brain Res Brain Res Rev, 37, 301-312.

[71] Van De Wiel, N.M., Van Goozen, S.H., Matthys, W., Snoek, H. \& Van Engeland, H. (2004). Cortisol and treatment effect in children with disruptive behavior disorders: a preliminary study. J Am Acad Child Adolesc Psychiatry, 43, 1011-1018.

[72] Wang, L.J., Hsiao, C.C., Huang, Y.S., Chiang, Y.L., Ree, S.C., Chen, Y.C., et al. (2011a). Association of salivary dehydroepiandrosterone levels and symptoms in patients 
with attention deficit hyperactivity disorder during six months of treatment with methylphenidate. Psychoneuroendocrinology, 36, 1209-1216.

[73] Wang, L.J., Huang, Y.S., Hsiao, C.C., Chiang, Y.L., Wu, C.C., Shang, Z.Y., et al. (2011b). Salivary dehydroepiandrosterone, but not cortisol, is associated with attention deficit hyperactivity disorder. World J Biol Psychiatry, 12, 99-109.

[74] Wang, L.J., Huang, Y.S., Hsiao, C.C., Chen, C.K. (2012). The Trend in Morning Levels of Salivary Cortisol in Children with ADHD during 6 Months of Methylphenidate Treatment. J Attention Disorders, (In press)

[75] Weizman, R., Dick, J., Gil-Ad, I., Weitz, R., Tyano, S. \& Laron, Z. (1987). Effects of acute and chronic methylphenidate administration on beta-endorphin, growth hormone, prolactin and cortisol in children with attention deficit disorder and hyperactivity. Life Sci, 40, 2247-2252.

[76] Wilens, T.E. (2008). Effects of methylphenidate on the catecholaminergic system in attention-deficit/hyperactivity disorder. J Clin Psychopharmacol, 28, S46-53.

[77] Wolf, O.T. \& Kirschbaum, C. (1999). Actions of dehydroepiandrosterone and its sulfate in the central nervous system: effects on cognition and emotion in animals and humans. Brain Res Brain Res Rev, 30, 264-288.

[78] Wolf, O.T., Neumann, O., Hellhammer, D.H., Geiben, A.C., Strasburger, C.J., Dressendorfer, R.A., et al. (1997). Effects of a two-week physiological dehydroepiandrosterone substitution on cognitive performance and well-being in healthy elderly women and men. J Clin Endocrinol Metab, 82, 2363-2367.

[79] Wolkowitz, O.M., Reus, V.I., Keebler, A., Nelson, N., Friedland, M., Brizendine, L., et al. (1999). Double-blind treatment of major depression with dehydroepiandrosterone. Am J Psychiatry, 156, 646-649.

[80] Yang, S.J., Shin, D.W., Noh, K.S. \& Stein, M.A. (2007). Cortisol is inversely correlated with aggression for those boys with attention deficit hyperactivity disorder who retain their reactivity to stress. Psychiatry Res, 153, 55-60.

[81] Young, R., Sweeting, H. \& West, P. (2012). Associations between DSM-IV diagnosis, psychiatric symptoms and morning cortisol levels in a community sample of adolescents. Soc Psychiatry Psychiatr Epidemiol, 47, 723-733.

[82] Yu, Y.Z. \& Shi, J.X. (2009). Relationship between levels of testosterone and cortisol in saliva and aggressive behaviors of adolescents. Biomed Environ Sci, 22, 44-49. 